\title{
The Effect of Experience and Embodiment on Empathetic Behavior toward Virtual Agents
}

\author{
Yuna Kano* \\ Junya Morita* \\ kano.yuhna.15@shizuoka.ac.jp \\ j-morita@inf.shizuoka.ac.jp \\ Shizuoka University \\ Hamamatsu, Shizuoka, Japan
}

\begin{abstract}
To realize a harmonized relationship between human and computational systems, it is necessary to understand the nature of empathy for a virtual agent and the kinds of behaviors elicited by such mental activity. By setting a ball-tossing game in which a participant and two virtual agents were involved, we examined how ostracism experiences affect cognitive empathy and how embodiment induced by VR (Virtual Reality) influences behaviors that elicit emotional empathy. Thirty-two participants were divided into two groups by manipulating the experience of being ostracized in the preparatory task. In the main task, the participants played the game under two conditions: an equitable condition, in which the virtual agents tossed the ball evenly to the participant and the other agent, and an inequitable condition, in which one agent tossed the ball only to the participant. The data obtained in the experiment was analyzed from the viewpoint of helping behaviors to the agent who was ostracized and examined the relation of this behavior with the EQ (Empathy Quotient), an index to measure an individual's empathetic traits. As a result, the ostracized experience made the participants notice the ostracizing behaviors of the agent and fostered helping behavior toward the agent who was ostracized. In addition, the participants with a high EQ tended not to notice the ostracizing behavior in the situation without embodiment induced by VR, whereas they exhibited more helping behaviors when they experienced the task with the VR device. We consider that these findings contribute to the design of smoother interaction between humans and virtual agents.
\end{abstract}

\section{CCS CONCEPTS}

- Human-centered computing $\rightarrow$ HCI theory, concepts and models.

\section{KEYWORDS}

Empathy; (the) Empathy quotient; Embodiment; VR; (the) Cyberball game

"Both authors contributed equally to this research.

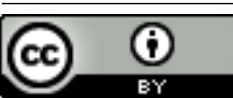

This work is licensed under a Creative Commons Attribution International 4.0 License.

HAI '20, November 10-13, 2020, Virtual Event, NSW, Australia

(C) 2020 Copyright held by the owner/author(s)

ACM ISBN 978-1-4503-8054-6/20/11.

https://doi.org/10.1145/3406499.3415074
ACM Reference Format:

Yuna Kano and Junya Morita. 2020. The Effect of Experience and Embodiment on Empathetic Behavior toward Virtual Agents. In Proceedings of the 8th International Conference on Human-Agent Interaction (HAI '20), November 10-13, 2020, Virtual Event, NSW, Australia. ACM, New York, NY, USA, 9 pages. https://doi.org/10.1145/3406499.3415074

\section{INTRODUCTION}

Information technology is rapidly evolving, and the role of computers is changing from simply carrying out tasks to living together with humans while supporting them. In the field of HumanAgent Interaction (HAI), such relationships have been studied for many years. For example, Nass et al. [15] pointed out that the social rules governing Human-Human Interaction (HHI) can apply equally to Human-Computer Interaction (HCI). The same researchers [16] also proposed a concept of media equation claiming that humans tend to treat a communication medium, namely an agent, as a human.

In the current study, the authors focus on empathy as a mental function that enables in the natural and smooth HCI. Empathy generally plays a vital role in HHI. Furthermore, the authors believe that this mental ability has evolved to maintain harmony in human society, allowing people to comprehend emotions from another's perspective. Therefore, achieving empathetic relations between humans and computational systems could facilitate more natural HCI.

To realize such a new relationship between human and computational systems, it is necessary to understand both the nature of empathy in a computational agent and the kinds of behaviors elicited by such mental activity. To approach these problems, we first assume a diverse concept of empathy, considering that there are at least two different components of abilities: an emotional component, which elicits emotional responses from other people, and a cognitive component, which captures the other person's perspective. These concepts are generally agreed on by researchers dealing with human empathy $[2,5]$, and we consider that this discussion can also apply to human empathy for a computational agent (similar discussions are in [21]). We then identify the factors that enhance each component of empathy, having manipulated these in experiments in which participants interacted with virtual agents.

As a factor enhancing cognitive empathy, we focus on the use of past experience. This process involves perspective-taking, which can be considered an analogy [9] that connects one's past experiences (base) to those of others in the current situation (target). To 
make such an analogy, an appropriate base experience is necessary. In fact, past studies have shown that empathy is difficult for individuals who lack the base of perspective [7, 11]. To control for this, the current study creates the experience of ostracism in the laboratory to draw out empathetic behavior toward an agent.

The embodiment aspect of VR (Virtual Reality) can also be assumed to be a factor affecting human empathy. VR is a technology that gives a user an artificially constructed sense of reality. Recently, VR devices such as HMD (Head-Mounted Display) and physical controllers have allowed people to experience immersion in digitally created three-dimensional (3D) worlds. Interaction with agents using such technology provides a qualitatively different experience from using a normal pointing device and a flat display due to the user's sense of embodiment. In such a VR situation, humans' primitive emotions are assumed to be strongly evoked, leading to the emotional experience of empathy through a process of emotional contagion [10].

To observe a behavior induced by these two factors, this paper presents an experiment in which participants could choose to ostracize one of two agents. It then examines the relationship between this behavior and emotional ability as measured by the empathy quotient (EQ) [2]. Before presenting the experiment, we explain the purpose of this study within the context of relevant past research. Following this, participants' behaviors and subjective impressions in the experiment are presented. Finally, the results of the experiment are discussed in reference to the element of empathy.

\section{PREVIOUS RESEARCH AND ITS PURPOSE}

In this section, we explain the previous studies that we consulted to build our experiment on empathic relationships in HAI. Based on these studies' findings, we clarify the study purpose.

\subsection{Empathy ability}

Several researchers have proposed measures of empathy by defining the components of this ability. One of the most frequently utilized measures is the Interpersonal Reactivity Index (IRI) proposed by Davis [4]. This is a multi-dimensional scale of empathic traits, including four scales: Empathic Concern, Perspective Taking, Personal Distress and Fantasy Scale. These sub-scales were selected according to the broadest meaning of empathy, namely the reaction of one individual to the observed experience of another. However, it is not clear how these scales are related to each other or what types of empathetic behaviors are introduced by these components.

The other measure of empathy is the Empathy Quotient (EQ) developed by Baron-Cohen [2]. This index is based on the empathizingsystemizing (E-S) theory of individual differences caused by the general biological mechanism behind gender differences and mental disorders such as the autism spectrum disorder (ASD). It classifies individuals based on their abilities in empathic thinking (E) and systematic thinking (S), and measures skills using an Empathy Quotient (EQ) and Systemizing Quotient (SQ). These factors are related to the physiological processes caused by hormone activity (e.g., oxytocin), and are ultimately connected to the human evolutionary process. In this theory, empathy is assumed to be a complex combination of multiple elements, including emotional, cognitive, and mixed components. Emotional elements comprise the eliciting of appropriate emotions by seeing or knowing another's response, while cognitive elements involve understanding or predicting what others think, feel, or do. Contrary to IRI, EQ posits that these components are closely related and difficult to treat separately.

In this study, we adopted the view of empathy foundational to EQ, defining it as a mixed compound of cognitive and emotional processes generated by biological mechanisms, which occur in interpersonal relationships. The biological foundations of EQ might be useful to explain how the primitive parts of human functions relate to empathy for a computational agent. In this study, we specifically examined the kind of empathetic behavior involved in such interactions by linking EQ as an individual user trait to empathic behaviors.

\subsection{Experimental tasks to examine emotions in interpersonal situations}

As discussed in the previous subsection, empathy can be considered a mental process that occurs in interpersonal relationships and affects interactions with others. To carry out empirical research associated with empathy, we needed to define empathic behaviors through an experiment in which interpersonal emotion occurs.

Exploring a situation in which interpersonal emotion occurs, this study focused on ostracism, the exclusion of a group or individual. Cyberball [19] is an electronic game methodology widely used to study interpersonal ostracism and acceptance. This virtual ball-tossing game involves three players: one participant and two agents who simulate real players but are computer-generated and controlled. In Williams et al. [20]'s original experiment, half of the study participants received tosses equally from either agent, while the other half did not receive any tosses after tossing several times and were ostracized by the agents for five minutes. The researchers found that the ostracized participants reported lower credibility towards others, such as difficulty in working with them, as measured by a questionnaire administered after the game task.

Furthermore, a study using functional MRI [6] observed the blood oxygenation-dependent responses (BOLD) in the brains of the participants who played Cyberball. In the experiment, the activity of the anterior cingulate cortex, a region of the brain relating to physical pain, was activated when participants were ostracized. Notably, the same result was obtained even when the participant knew that the other players were computer-controlled agents. Other researchers have reported similar results in a 3-D virtual environment [14].

In addition to these social psychological studies, various HAI researchers have conducted experiments in which multiple agents/robots and human participants interact. Yoshikawa et al. [22] showed that human interaction with multiple robots improves participants' impression of robots more than one-on-one interaction with them. Other research has investigated an interactive design in which humans are free to participate in conversations with multiple robots [13]. These studies suggest that humans perceive their interaction 
with robots analogously to that which would occur in a human social setting, conducting relationships with them, and responding to robot behaviors

Summing up the above, the current study set an experimental task involving a participant and multiple virtual agents. Specifically, we extended Cyberball to allow participants to choose whether to ostracize or help an agent who was ostracized by another agent. In this situation, helping behavior toward the ostracized agent could be regarded as empathic behavior.

\subsection{Similar experience and empathy}

In the first part of this section, we defined empathy as a mixture of cognitive and emotional processes. Following this, we introduced our experimental setting in which an example of participants' empathic behavior could be observed, such as helping behavior toward an ostracized agent. We next need to clarify what factors influence such a mental process and associated behaviors.

One factor can be found in the literature about the effects of similar experiences on the empathetic process. For example, Hodge et al. [11] showed that participants without an experience like the target situation had difficulty in taking on the perspective of a target. Other experiments found a correlation between the degree of empathy experienced while reading a story and having had a similar experience as its characters [7]. This study demonstrated that similar experiences play an important role in feeling empathy for others.

We consider that the effects of similar experiences relate to cognitive empathy involving perspective-taking. In our study, to observe empathetic behavior toward an agent who is ostracized, we assumed that the experience in which a participant is ostracized by an agent functions as a base experience and induces empathetic behavior.

\subsection{VR and empathy}

Another focus in the literature has been the usage of the VR experience in experiments to verify compassion towards others. In one experiment [1], participants showed empathy toward the colorblind after experiencing color-blindness in VR. In other words, the experience of color blindness in VR intensified the reality of colorblindness such that subsequent empathy for real color-blindness was naturally induced.

In another study by the same authors, participants were given Superman-like flying abilities in VR and experienced helping actions. This elicited real-world empathetic behavior toward humans [17]. Participants could extend their arms to control flight, so the physical experience (embodiment) of actually moving the body played a role. In other words, performing a helping action with VR-facilitated embodiment led to the real-world helping action.

A theory proposed by de Waal [5] explains how embodiment affects empathy. In this theory, empathy consists of multiple structures set within an evolutionary perspective. The most primitive structure is physical perception, an action common to all animals that forms the foundation on which emotional empathy is overlaid. Furthermore, the theory supposes that emotional empathy leads to sympathy and cognitive empathy, which only human beings possess. It is thought that the introduction through VR of the primitive embodiment common to animals affects the primitive element of empathy, namely emotional empathy. Therefore, in our study, we postulate that emotional empathy is reflected in behavior from embodiment by VR.

\subsection{Study aims}

So far, we define empathy as a mixed compound of emotional and cognitive processes and assume certain factors that influence this process. In this study, we theorized that similar experience is related to cognitive empathy, embodiment by VR is related to emotional empathy, and that they incur empathic behavior. In the following description and analysis of the experiment, we examine the effect of such factors on empathy-related behavior toward a virtual agent and the relation with the individual empathic trait. Throughout this study, we aimed to reveal what factors draw out human empathic behavior towards a virtual agent.

\section{EXPERIMENT}

In this experiment, we analyzed empathic behavior in a modified Cyberball game involving a participant and multiple virtual agent. In this task, we examined how the ostracizing experience affects perspective-taking and how embodiment through VR influences behavior as an element that elicits emotional empathy. We will also examined the relationship between the individual trait of human empathy (EQ) and empathic behavior towards virtual agents.

\subsection{Method}

3.1.1 Participants. A total of 32 undergraduate students (18 males and 14 females) majoring in informatics participated in the experiment. Participants were compensated 1,500 yen for their participation. We divided participants into two groups (ostracized vs. nonostracized). In EQ measurement results, we did not find a significant difference between the ostrcised (mean $E Q=35.5, S D=8.90$ ) and the non-ostracized group (mean $E Q=35.3, S D=8.57$ ) (the procedures of measuring EQ is described below).

3.1.2 Experimental task. In this study, we constructed a task based on a modified Cyberball game [19]. In the original task, a participant is assigned the role of a player who is ostracized by two agents. In contrast, participants in our study decided for themselves whether to ostracize an agent or not. Specifically, we set it up so that one agent (Agent 2) throws the ball only to the participant and does not throw it to the other agent (Agent 1). If the participant returns the ball to Agent 2 only, Agent 1 will be ostracized in the field. On the other hand, if the participant throws the ball to Agent 1, the situation becomes cooperative among the three players. Within this scenario, we analyze empathetic behavior toward virtual agents as the helping behavior toward Agent 1, who is ostracized by Agent 2, and observe what cognitive and emotional contexts trigger the individual trait of empathy.

As a tool to implement the task, we used the game engine Unity [18], which enables various interactive environments, and the 3-D headset HTC Vive [12], which consists of an HMD headset, controllers, and a base station for tracking users' motions. Figure 1 
presents an image of a participant performing this task. The screenshot of the task developed with Unity is presented in Figure 2, where the simple two agents (the green and blue ones) stand in front of the participant. To prevent the appearance of the agents from affecting the results, we employed agents with an abstract appearance.

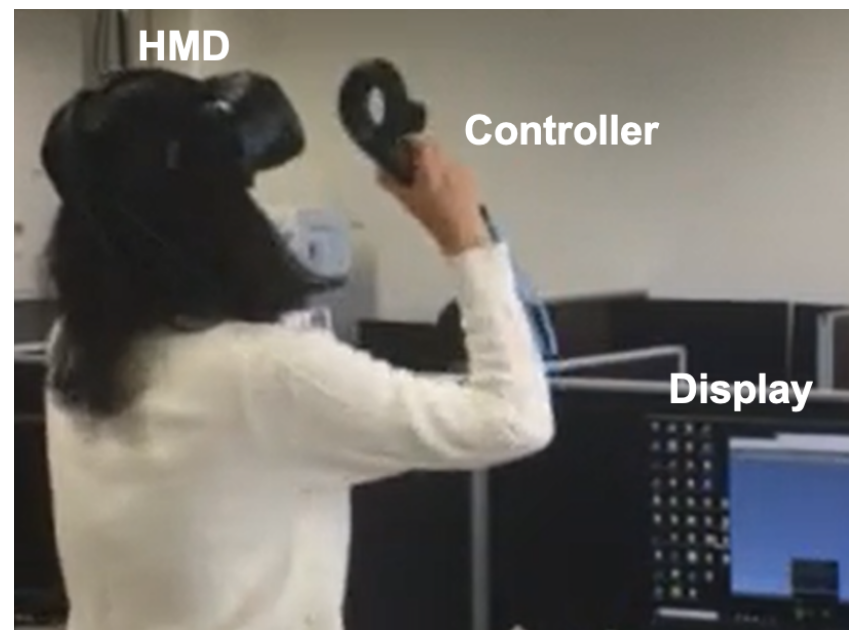

Figure 1: Image of a participant performing the game task. The display in the right-bottom shows the game scene that the participant observes.

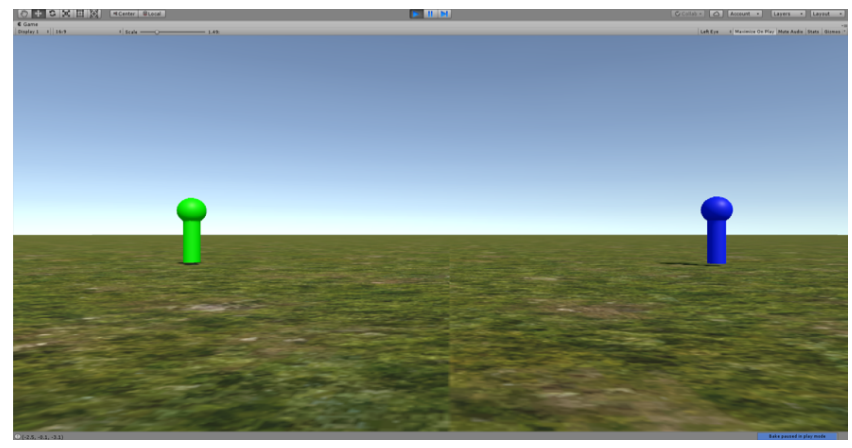

Figure 2: Game task screen

Both agents' behavior was designed to catch the ball directly thrown at it. To determine whether the ball was directed to the agent, we designed an algorithm to follow and pick up the ball at a range separated by four virtual world coordinates from the coordinates where the agent was located. When the ball failed to enter the range of either agent, the ball rolled on the ground while no agent caught it, and the participants had to throw it again.

3.1.3 Experimental design. To analyze the effect of similar experience on empathetic behavior, we divided participants into two groups (ostracized vs. non-ostracized) by manipulating the ostracism experience in a preparatory task in which participants experienced the ostracized role as in the original Cyberball game. Half of the participants $(n=16)$ engaged in this task, while the others $(n=$ 16) did not. We also controlled for four within-participant factors: mouse vs. Vive operation method, and equitable vs. inequitable agent behavior, as shown in Table 1. The comparison of operation methods allowed us to analyze the embodiment effect induced by VR. In the Vive condition, participants observed the agent in the 3-D environment and tossed the virtual ball by moving their arms. Also, the HMD headset allowed them to move their heads to redirect their perspective. In contrast, the mouse condition involved the observation of the agent in a flat display, using a mouse to choose the agent receiving the ball. These conditions allowed us to explore factors influencing empathetic behavior toward the ostracized agent. We examined the tendency of a participant to toss the ball to the ostracized agent under each condition and then calculated the correlation between this factor and EQ as the measure of empathetic traits.

The order of the above four within-participant factors, and the combination of the color (blue vs. green) and position (left vs. right) of the ostracized agent (Agent 1) were counterbalanced, yielding a total of 16 possible combinations. The 16 participants in each group participated in different counterbalanced conditions. Figure 3 shows an example of the experimental conditions.
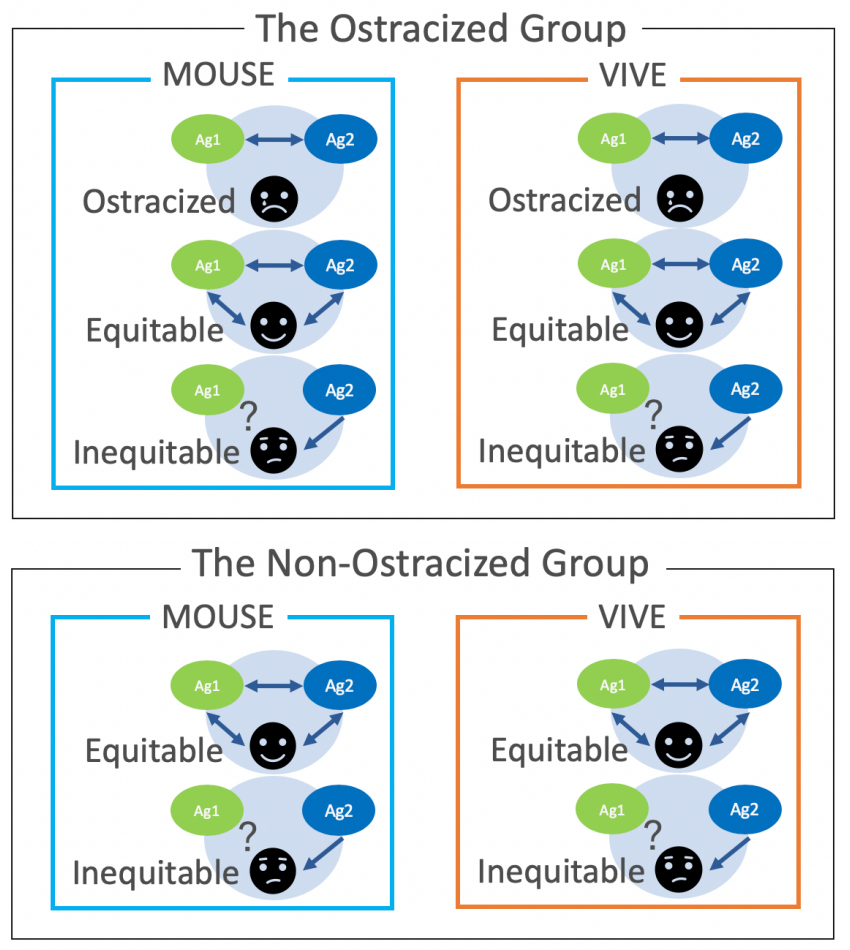

Figure 3: An example of the experimental conditions; the order of conditions in each group was counter-balanced among the participants.

3.1.4 Procedure. After an explanation of the experiment, the participants were instructed about the rules of the game task and operation of the devices for each method (mouse vs. HTC Vive). The 
Table 1: Experimental Conditions

\begin{tabular}{|c|c|c|c|}
\hline & \multicolumn{2}{|c|}{ Operation method } \\
\hline & & Mouse condition & Vive condition \\
\hline \multirow[b]{2}{*}{$\begin{array}{l}\text { Agent behavior } \\
\text { tendency }\end{array}$} & $\begin{array}{l}\text { Equitable } \\
\text { condition }\end{array}$ & $\begin{array}{l}\text { Play Cyberball with mouse and keyboard. Both } \\
\text { agents randomly toss the ball to the participant } \\
\text { and the other agent. }\end{array}$ & $\begin{array}{l}\text { Play Cyberball with HTC Vive. Both agents } \\
\text { randomly toss the ball to the participant and the } \\
\text { other agent. }\end{array}$ \\
\hline & $\begin{array}{l}\text { Inequitable } \\
\text { condition }\end{array}$ & $\begin{array}{l}\text { Play Cyberball with mouse and keyboard. When } \\
\text { the ball is tossed by Agent } 1 \text {, it goes to a random } \\
\text { opponent. When the ball is tossed by Agent 2, it } \\
\text { goes only to the participants. }\end{array}$ & $\begin{array}{l}\text { Play Cyberball with HTC Vive. When the ball is } \\
\text { tossed by Agent 1, it goes to a random opponent. } \\
\text { When the ball is tossed by Agent 2, it goes only } \\
\text { to the participants. }\end{array}$ \\
\hline
\end{tabular}

task was presented as a ball-tossing game involving three players, and the participants performed a test play before each operational condition started. In the test play, the participants tossed the ball to one agent with the mouse or the HTC Vive.

The main task consisted of four sessions in which the operation methods and agent behavior tendencies varied. Each session lasted five minutes. In the preparatory tasks, the participants in the ostracized group played a game in an equitable way for one minute; thereafter, they did not receive the ball at all from either agent for four minutes.

After the main task, the participants were administered the postquestionnaire and psychological tests measuring individual traits, including the Japanese version of Baron-Cohen's EQ and SQ indices [2]. The post-questionnaire checked whether the participants were aware of the difference in agent behavior tendency between the equitable and the inequitable conditions. First, they answered a binary choice question that asked whether they felt the differences between the equitable and inequitable conditions of each operation method (mouse vs. HTC Vive). Following this, the participants who answered "yes" to the above question freely wrote about what differences they noticed. EQ and SQ were measured at the end of the experiment to prevent the participants from noticing the experimental aim beforehand, thereby avoiding any influence on their behaviors during the experiment.

3.1.5 Acquired data. Summarizing the above, we obtained data consisting of the operation logs during the main task and the results of post-questionnaires. The former data recorded the timing of the ball-tossing and the $\mathrm{x}-\mathrm{y}$ coordinates of each player. From these, the following indicators were extracted.

- Ag1: the number of tosses toward Agent 1

- Ag2: the number of tosses toward Agent 2

- Ag1/Ag2: the ratio of tosses toward agents

Among them, we focused on $A g 1 / A g 2$ as the index of the empathetic behavior toward the ostracized agent. In the inequitable condition, Agent2 tossed the ball only to the participant. So, the small $A g 1 / A g 2$ indicates that the participant tossed the ball exclusively to Agent 2 and ostracized Agent 1 . Throughout the following analysis, we adopt a significance level of 0.05 and a marginally significant level of 0.10 .
Table 2: The mean number of tosses for each condition; the numbers in the parentheses indicate the standard deviations.

\begin{tabular}{|c|c|c|c|c|}
\hline \multirow{2}{*}{} & \multicolumn{2}{|c|}{$\begin{array}{c}\text { Ostracized } \\
\text { participant }\end{array}$} & \multicolumn{2}{c|}{$\begin{array}{c}\text { Non-ostracized } \\
\text { participant }\end{array}$} \\
\cline { 2 - 5 } & $A g 1$ & $A g 2$ & $A g 1$ & $A g 2$ \\
\hline M(Equitable) & $14.5(3.6)$ & $15.1(2.6)$ & $14.8(3.4)$ & $15.8(4.1)$ \\
M(Inequitable) & $23.1(3.3)$ & $15.4(4.1)$ & $20.9(3.3)$ & $19.1(4.8)$ \\
V(Equitable) & $13.5(4.3)$ & $15.6(2.7)$ & $15.3(3.7)$ & $14.8(3.0)$ \\
V(Inequitable) & $22.5(6.1)$ & $15.2(3.1)$ & $21.5(5.2)$ & $16.2(4.2)$ \\
\hline
\end{tabular}

\subsection{Results and discussion}

3.2.1 Comparison of Ag1/Ag2 under each condition. Table 2 presents the mean number of tosses for each condition. From these data, we calculated the mean of $A g 1 / A g 2$ in each experimental condition (Figure 4). Participants who scored below 1.0 were more likely to toss to Agent 2, while those who scored above 1.0 tossed more to Agent 1. Using this index as a dependent variable, we performed a three-way [ostracized experience (between participants) $\mathrm{x}$ operation method (within-participant) $\mathrm{x}$ agent behavior tendency (within-participant)] analysis of variance (ANOVA). The analysis revealed no significant second-order interaction $(F(1,30)=$ $0.56, p=.46)$ and a significant two-way interaction of the ostracized experience $\mathrm{x}$ the agent behavior tendency $(F(1,30)=6.86$, $p=.01)$. Following the above two-way interaction, the simple main effects of the ostracized experience were analyzed and showed a significant difference under the inequitable condition $(F(1,30)=$ $5.46, p=.02)$. However, no significant difference was evident under the equitable condition $(F(1,30)=1.39, p=.24)$. Also, concerning the simple main effect of the agent behavior tendency, we found a significant difference under the ostracized condition $(F(1,30)=29.24, p<.01)$ and a marginally significant difference under the non-ostracized condition $(F(1,30)=2.91, p=.09)$.

The results confirmed that participants who had been ostracized made more tosses to the ostracized agent (Agent 1 in the inequitable condition) than those who had not been ostracized. From this, we considered that the participant's ostracized experience elicited the participant's empathy for Agent 1 and led to the helping action for Agent 1. We posit that performing such empathetic behavior requires the ability of perspective-taking [2]. In the ostracized condition, the participant was first ostracized by two agents. Following this experience, s/he observed that Agent 1 was ostracized by Agent 2 under the inequitable conditions. Previous 
studies have pointed out that when a person has had a similar experience to the target, it will be possible to perform perspectivetaking $[7,11]$. From such discussions, we can interpret that the ostracized experience under the ostracized condition of this task was easily invoked as a similar experience because the ostracized experience was performed shortly before the inequitable condition in the same environment.

Therefore, the cognitive empathy of the participant was assumed to be elicited by the participant's ostracized experience, which was a similar experience to that of Agent 1, who had been ostracized. We considered that this led to the helping action for Agent 1.

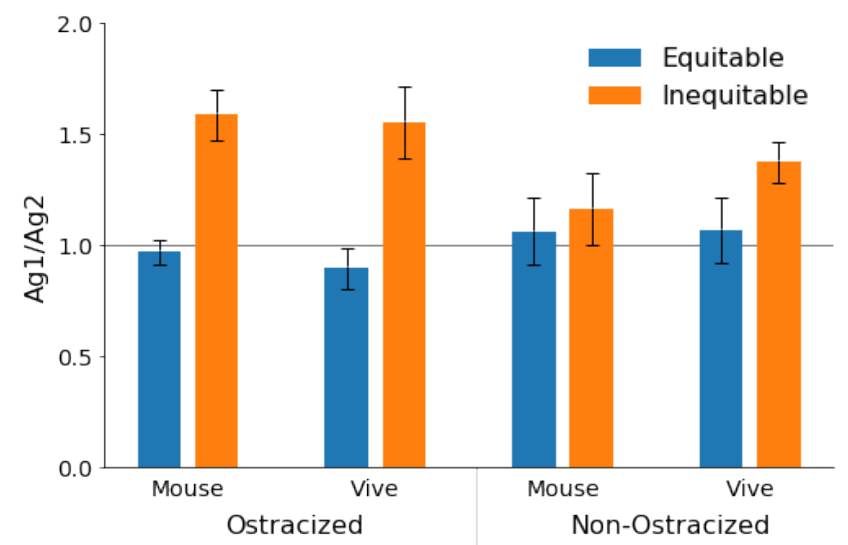

Figure 4: $A g 1 / A g 2$ to agents for each condition; error bars represent the standard error of means. Higher than the $\mathbf{1 . 0}$ line indicates more tosses to the ostracized Ag1.

3.2.2 Correlation between toss number and EQ in the game task. Table 3 shows the Spearman's rank correlation coefficients between the number of tosses for each agent and EQ under each condition. In the table, $\mathrm{M}$ indicates the mouse condition while $\mathrm{V}$ represents the Vive condition.

As can be seen, no significant correlation was found in the mouse equitable/inequitable conditions and the VR equitable condition of ostracized participants, and non-ostracized participants. On the other hand, for the ostracized participants under the VR and inequitable conditions, the EQ showed a positive correlation with the number of tosses made to the Agent $1\left(r_{s}=0.699, p<.01\right)$.

To confirm that the obtained correlation was specific to the empathy traits, we applied the same analysis to the SQ. Table 4 shows the Spearman's rank correlation coefficients between the number of tosses for each agent and SQ. No significant results were found here. Therefore, this effect is considered specific to EQ.

The above correlations do not indicate a strong relationship between individual human empathic traits and empathic behaviors toward virtual agents. However, it is suggestive to find that a significant correlation was only observed in the condition in which the experienced participants played a game with an embodied device. This result suggests that empathy toward an agent is associated with both the factors of experience and embodiment. Regarding the effect of experience, we expected that perspective-taking,
Table 3: Correlation Analysis between Toss Number and EQ in the Game Task

\begin{tabular}{|c|c|c|c|c|c|c|}
\hline \multirow{2}{*}{} & \multicolumn{3}{|c|}{$\begin{array}{c}\text { Ostracized } \\
\text { participant }\end{array}$} & \multicolumn{3}{c|}{$\begin{array}{c}\text { Non-ostracized } \\
\text { participant }\end{array}$} \\
\cline { 2 - 7 } & $A g 1$ & $A g 2$ & $A g 1 / A g 2$ & $A g 1$ & $A g 2$ & $A g 1 / A g 2$ \\
\hline $\mathrm{M}($ Equitable) & -0.108 & -0.092 & -0.088 & -0.087 & -0.124 & 0.264 \\
$\mathrm{M}($ Inequitable) & 0.057 & -0.188 & 0.063 & -0.232 & 0.200 & -0.227 \\
$\mathrm{~V}$ (Equitable) & 0.015 & 0.457 & -0.033 & -0.165 & 0.093 & -0.238 \\
$\mathrm{~V}$ (Inequitable) & $0.699^{*}$ & 0.319 & 0.407 & 0.171 & 0.363 & -0.264 \\
\hline
\end{tabular}

Table 4: Correlation Analysis between Toss Number and SQ in the Game Task

\begin{tabular}{|c|c|c|c|c|c|c|}
\hline & \multicolumn{3}{|c|}{$\begin{array}{c}\text { Ostracized } \\
\text { participant }\end{array}$} & \multicolumn{3}{c|}{$\begin{array}{c}\text { Non-ostracized } \\
\text { participant }\end{array}$} \\
\cline { 2 - 7 } & $A g 1$ & $A g 2$ & $A g 1 / A g 2$ & $A g 1$ & $A g 2$ & $A g 1 / A g 2$ \\
\hline $\mathrm{M}($ Equitable) & -0.220 & -0.146 & 0.098 & 0.303 & -0.027 & 0.113 \\
$\mathrm{M}$ (Inequitable) & 0.053 & 0.247 & -0.202 & 0.168 & -0.229 & 0.277 \\
$\mathrm{~V}$ (Equitable) & 0.014 & 0.167 & 0.011 & -0.272 & -0.348 & 0.234 \\
$\mathrm{~V}$ (Inequitable) & 0.005 & 0.085 & -0.062 & -0.112 & 0.030 & -0.202 \\
\hline
\end{tabular}

an element of cognitive empathy, requires a similar experience. Concerning embodiment, the embodied sensation is a primary element of empathy, as postulated by the theory of de Waal [5]. It is considered that the embodiment introduced in the Vive condition stimulated a primitive sensation and elicited empathetic behavior towards the agent. Based on this assumption, it can be considered that a high EQ score indicates greater access to primitive empathy.

However, the effect of EQ was only observed in participants who had been ostracized. This indicates that empathetic tendencies do not reflect only primitive behavior; rather, some cognitive ability based on past experience is needed in taking on perspectives toward the agent. These results suggest that cognitive empathy and emotional empathy cannot be separated; an interpretation consistent with the assertion made by Baron-Cohen [2] that empathy is inseparable from both emotional and cognitive components.

3.2.3 Analysis of the post-questionnaire. We analyzed the postquestionnaire to determine if participants perceived the agents' behavioral tendencies. Figure 5 indicates the number of participants who felt the difference in agent behaviors between the equitable and inequitable conditions. We performed chi-square tests for the two operation conditions and found that more participants in the ostracized group felt the difference than those in the nonostracized group (Mouse: $\chi^{2}(1)=4.99, p=.03$, Vive: $\chi^{2}(1)=8.29$, $p<.01)$.

We also examined the relation between feeling the difference and empathic traits. Figure 6 indicates the EQ of participants who felt the difference and those who did not. In this analysis, we summarized the ostracized and non-ostracized conditions, because of the ratio of participants who felt the difference versus those who did not feel it differed between the conditions. We performed a 2-way [operation method (between-participants) $\mathrm{x}$ felt the difference (between participants)] ANOVA, and found no significant interaction $(F(1,62)=1.68, p=.20)$, no significant main effect of the operation method $(F(1,62)=0.18, p=.67)$, and a marginally 
significant main effect of feeling the difference $(F(1,62)=2.84$, $p=.10)$.

In the mouse condition, direct comparisons between the participants who felt the difference versus those who did not show a significant difference, indicating that the participants who did not feel the difference had a high EQ as compared to those who felt the difference $(t(23)=2.27, p=.03)$. No significant difference was found in the Vive condition $(t(25)=0.27, p=.79)$

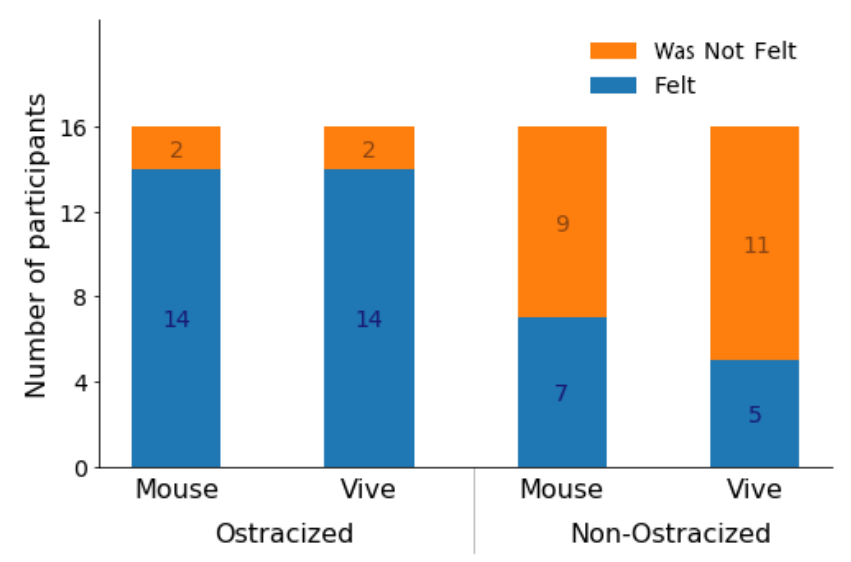

Figure 5: Number of participants who felt the difference between the equitable and inequitable conditions

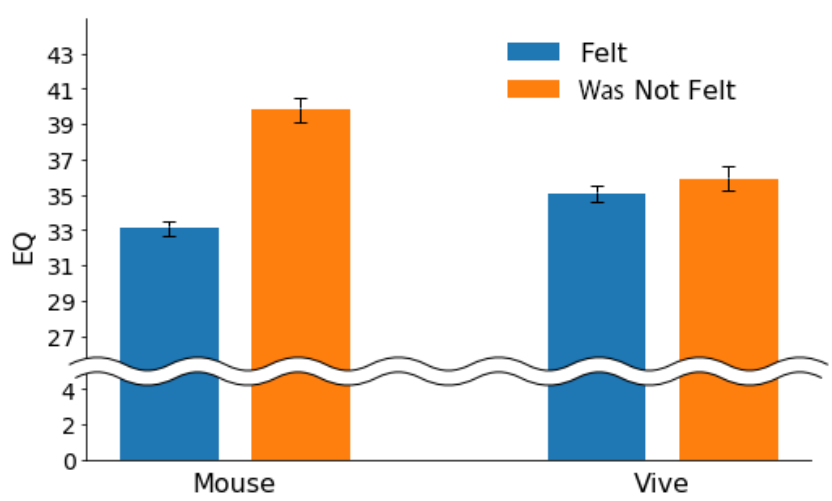

Figure 6: EQ of participants; error bars represent the standard error of means. The scale of the vertical axis was adjusted according to the variance of the means.

From the result indicated in Figure 5, we consider that the ostracized experience made the participants aware of the agents' behavioral changes. This interpretation supports the result in Section 3.2.1 where the participant's ostracized experience elicited empathetic behavior (helping the agent) toward the ostracized agent. In other words, it can be considered that the empathetic behavior observed in this experiment was at least partially induced by the cognitive component of empathy.
Contrary to the above discussion about the effect of experience, it is difficult to interpret the EQ-related results. Figure 6 indicates the participants with a high EQ had a tendency not to feel the behavioral changes between the equitable and inequitable conditions. This tendency was particularly strong in the mouse condition, and it was mitigated in the Vive condition. The result obtained here might be interpreted together with the result obtained in Section 3.2.2. In the previous analysis, we found that participants with a higher EQ in the ostracized group were more likely to toss the ball to Agent 1 in the Vive condition. Therefore, we speculate that in the mouse condition, in which the participants did not experience physical movement, their empathetic ability was not triggered to detect Agent 2's ostracizing behavior. Eventually, they did not help the ostracized Agent 1 . This interpretation is consistent with Bloom's against empathy theory, which focuses on the negative aspects of empathy [3]. According to him, empathy is $a$ spotlight directing attention, working on certain targets but not on an unspecified number of people. This property of empathy might make the participant focus on Agent 2, who more frequently tossed the ball to the participants. This discussion suggests that the role of embodiment by VR in this task triggers the emotional empathic traits and directs their attention towards the ostracized Agent 1. The embodiment by VR may turn the spotlight of empathy from Agent 2 to the ostracized Agent 1, making the person more sympathetic.

\section{CASE STUDY}

The above discussion suggests that empathetic behavior is not entirely a primitive action initiated through an unconscious process but appears to be an overt cognitive process as well. However, the data in the previous section did not depict the online thinking process in the task. Therefore, to support this suggestion and describe the process of empathy toward a virtual agent in more detail, we conducted a case study to record and analyze a think-aloud proto$\operatorname{col}[8]$.

\subsection{Method}

4.1.1 Participants. A male student majoring in informatics, who had not participated in the previous experiment, participated in this case study and was given 1,500 yen as compensation. He scored 41 in EQ, which was higher than 35.4-the mean of EQ in the experiment presented in Section 3.

4.1.2 Task and procedure. Using the think-aloud protocol, the procedures of the first experiment were again employed [8]. The participant was instructed to freely talk aloud to express what he thought during the task, and this verbalization was recorded using a video camera. The order of the within-participant factors was as follows: Vive ostracized condition, Vive inequitable condition, Vive equitable condition, mouse ostracized condition, mouse inequitable condition, and mouse equitable condition.

\subsection{Results and discussion}

Table 5 shows examples of the verbalizations of the participant under each condition. First, in the ostracized condition, both the mouse and the Vive conditions showed verbalization that expressed feelings of emotional pain caused by the ostracizing behavior of 
the agents. These indicated that in this experimental environment, negative emotions were aroused in the participant, who felt pain in being ostracized by the agent, as in the original Cyberball experiment [20].

Next, from the verbalizations under the inequitable condition, it is clear that participants noticed the behavior in which only Agent 2 tossed the ball to the participant (Verbalizations 6 and 8 in Table 5). In addition, Verbalization 10 in Table 5 shows that the participant thought about the relationship between the two agents. Furthermore, from Verbalization 7 in Table 5, it can be seen that the participant perceived the agent in this task as a human and felt sympathetic. These results suggest that the participants' helping behavior in the experiment was accompanied by the cognitive process, at least at the level reflected in the think-aloud protocol.

Table 5: Participant's utterance examples under each condition (translations from Japanese).

\begin{tabular}{ll}
\hline \hline \multirow{2}{*}{ (Ostracism) } & $\begin{array}{l}\text { (1)I'm sad. } \\
\text { (2)Being ostracized is an excruciat- } \\
\text { ing pain. }\end{array}$ \\
\hline \multirow{2}{*}{ (O) Ostracism) } & $\begin{array}{l}\text { (3)I'm sad. } \\
\text { (4)It felt empty. } \\
\text { (5)I'm really sad with just VR. }\end{array}$ \\
\hline & $\begin{array}{l}\text { (6)Blue only tosses it to me. } \\
\text { (7)At the moment of throwing, } \\
\text { it looks like an agent is looking to me. }\end{array}$ \\
\hline & $\begin{array}{l}\text { (8)Green tosses it to blue. } \\
\text { (9)Blue doesn't toss it to green. } \\
\text { (10)Do blue and green hate each } \\
\text { other? Or does blue one-sidedly } \\
\text { hate green? }\end{array}$ \\
\hline
\end{tabular}

\section{GENERAL DISCUSSION}

In the experiment, it was discovered that the experience of being ostracized by agents made participants perceive the ostracizing agent's behavior and fostered helping behavior directed toward the ostracized agent. This suggests that empathetic behavior for a virtual agent is based on perspective-taking, in which one maps one's own position, based on past experience, onto the other's position in the current situation. Therefore, it appears that the ostracized experience of the participants fosters the cognitive element of empathy for a virtual agent.

In addition to the ostracized experience, the embodiment induced by VR fostered the helping behavior of the high EQ participants toward the agent who was ostracized by the other agent. In the mouse condition, the participants with a high EQ tended not to notice the ostracizing behavior. In contrast, they performed more helping behaviors when they experienced the task with the VR device. From this result, we believe that a realistic embodiment is necessary to elicit the emotional empathic trait toward a virtual agent.

Our case study also confirms the role of the overt cognitive element of empathy for a virtual agent. In the case study, the online think-aloud protocol of the participant was recorded. Since the think-aloud protocol is assumed to reflect conscious behavior [8], the fact that a participant's verbalization included sympathy for the agent indicates that the empathetic behavior of the participant was not purely a function of unconscious emotional contagion but involves a conscious cognitive process.

We also examined the relation between empathetic behavior for a virtual agent and EQ, the individual trait of human empathy. According to Baron-Cohen, EQ measures a complex combination reflecting both the emotional and cognitive elements of empathy [2]. However, in this study, EQ did not have direct effects on empathetic behaviors. This index only influenced the helping behavior in the Vive ostracized condition, in which strong embodiment and cognitive cues of empathy were introduced. Interestingly, this index also negatively affected the perception of ostracizing behavior in the mouse condition. Such results suggest the negative side of human empathy towards virtual agents [3]. Further investigation is needed to confirm the detailed relationship between these two elements of empathy.

There are several other unanswered questions in this research. Compared to the factor of experience, the factor of embodiment did not have a strong effect on empathic behaviors. The factor of embodiment only affected high EQ participants. This result suggests that the possession of similar experience is a precondition of empathy. In fact, past studies showing the effect of VR [1, 14] also relied on the existence of similar participant experiences. To explore the relationship between experience and embodiment, it is necessary to conduct further research to manipulate the strength of embodiment and the structure of a similar experience.

The result of this study would be strengthened by conducting larger experiments. The experiment adopted a within-subject design on the manipulation of embodiment factors. Although the order of such factors was counterbalanced, this procedure can affect results; the participant may feel it is challenging to distinguish agents across the session, which could lead to the small effect of the operation methods.

The verbal protocols in the case study also need to be statistically analyzed. If we had collected more data, we could have examined the relationship between such an overt verbalization of sympathy with the experimental condition, individual empathic traits, and observed empathic behaviors. We can also conduct a sentiment analysis using a technique of natural language processing concerning these variables.

\section{CONCLUSION}

In this study, to examine factors enabling natural and smooth HAI, we sought to reveal what factors draw out human empathetic behavior toward an agent. The findings indicate that both experience and embodiment are important factors in eliciting human empathetic behavior. We also posit that emotional and cognitive abilities enable the effects of these two factors, and we confirmed that these two factors affected participants' behavior toward an agent. We believe that our results can contribute to the design of smoother HAI, noting the importance of the experience and embodiment. However, further research is needed to examine whether the same result is obtained in tasks involving mechanisms other than the ball-tossing task. 


\section{REFERENCES}

[1] Sun Joo Ahn, Amanda Minh Tran Le, and Jeremy Bailenson. The effect of embodied experiences on self-other merging, attitude, and helping behavior. Media Psychology, 16(1):7-38, 2013.

[2] Simon Baron-Cohen. The essential difference: Male and female brains and the truth about autism (M.Miyake, Trans.). Basic Books (Original work published 1814), 2005.

[3] Paul Bloom. Against empathy: The case for rational compassion. Random House, 2017

[4] Mark H Davis. Measuring individual differences in empathy: Evidence for a multidimensional approach. Fournal of personality and social psychology, 44(1):113, 1983.

[5] Frans de Waal. The age of empathy: Nature's lessons for a kinder society. Broadway Books, 2010.

[6] Naomi I Eisenberger, Matthew D Lieberman, and Kipling D Williams. Does rejection hurt? An fMRI study of social exclusion. Science, 302(5643):290-292, 2003.

[7] Jakob Eklund, Teresia Andersson-Stråberg, and Eric M Hansen. "I’ve also experienced loss and fear": Effects of prior similar experience on empathy. Scandinavian journal of psychology, 50(1):65-69, 2009.

[8] K Anders Ericsson and Herbert A Simon. Verbal reports as data. Psychological Review, 87(3):215, 1980.

[9] Dedre Gentner. Structure-mapping: A theoretical framework for analogy. Cognitive Science, 7(2):155-170, 1983.

[10] Elaine Hatfield, John T Cacioppo, and Richard L Rapson. Emotional contagion Current directions in psychological science, 2(3):96-100, 1993

[11] Sara D Hodges, Kristi J Kiel, Adam DI Kramer, Darya Veach, and B Renee Villanueva. Giving birth to empathy: The effects of similar experience on empathic accuracy, empathic concern, and perceived empathy. Personality and Social Psychology Bulletin, 36(3):398-409, 2010.
[12] HTC. Vive user guide, 2016

[13] Takamasa Iio, Yuichiro Yoshikawa, and Hiroshi Ishiguro. Retaining humanrobots conversation: Comparing single robot to multiple robots in a real event. Fournal of Advanced Computational Intelligence and Intelligent Informatics, 21(4):675-685, 2017.

[14] Oswald D Kothgassner, Johannna Xenia Kafka, Johanna Rudyk, Leon Beutl, Helmut Hlavacs, and Anna Felnhofer. Does social exclusion hurt virtually like it hurts in real-life? the role of agency and social presence in the perception and experience of social exclusion. Proceedings of the International Society for Presence Research, 15:45-56, 2014.

[15] Clifford Nass, Jonathan Steuer, and Ellen R Tauber. Computers are social actors. In Proceedings of the SIGCHI conference on Human factors in computing systems, pages $72-78,1994$.

[16] Byron Reeves and Clifford Ivar Nass. The media equation: How people treat computers, television, and new media like real people and places. Cambridge university press, 1996.

[17] Robin S Rosenberg, Shawnee L Baughman, and Jeremy N Bailenson. Virtual superheroes: Using superpowers in virtual reality to encourage prosocial behavior PloS one, 8(1):e55003, 2013.

[18] Unity. Unity User Manual 2018.2, 2018.

[19] Kipling D Williams and Blair Jarvis. Cyberball: A program for use in research on interpersonal ostracism and acceptance. Behavior research methods, 38(1):174180, 2006.

[20] Kipling D Williams and Kristin L Sommer. Social ostracism by coworkers: Does rejection lead to loafing or compensation? Personality and Social Psychology Bulletin, 23(7):693-706, 1997.

[21] Ozge Nilay Yalcin and Steve DiPaola. A computational model of empathy for interactive agents. Biologically inspired cognitive architectures, 26:20-25, 2018.

[22] Soshi Yoshikawa, Kataras Nihan, Mishio Okada, Keiko Shimazaki, Chihiro Sannomiya, and Kazunari Nawa. A social robot platform for personalized interactive companions. Robot Design Competition, 2016. 